\title{
EXISTENCE OF SOLUTIONS FOR SECOND-ORDER EVOLUTION INCLUSIONS
}

\author{
NIKOLAOS S. PAPAGEORGIOU ${ }^{1}$ \\ National Technical University, Department of Mathematics \\ Zografou Campus, Athens 15773, GREECE
}

(Received August, 1994; revised November, 1994)

\begin{abstract}
In this paper we examine second-order nonlinear evolution inclusions and prove two existence theorems; one with a convex-valued orientor field and the other with a nonconvex-valued field. An example of a hyperbolic partial differential inclusion is also presented.

Key words: Evolution Triple, Monotone Operator, Hemicontinuous Operator, Symmetric Operator, Fixed Point, Sobolev Space, Program, Average Turnpike Property, Separation Theorem.
\end{abstract}

AMS subject classification: $34 \mathrm{G} 20$.

\section{Introduction}

In this paper we study the existence of solutions for second order nonlinear evolution inclusions. Our work here complements the existence results of [7], where we considered first order nonlinear evolution inclusions. We present two existence results. One in which the multivalued term (orientor field) is convex valued and the other with a nonconvex valued orientor field. At the end of the paper, we work in detail an example of a hyperbolic partial differential inclusion, illustrating the applicability of our result.

\section{Mathematical Preliminaries}

Let $T=[0, r]$ and $Y$ a separable Banach space. Throughout this paper we will be using the following notation: $P_{f(c)}(Y)=\{A \subseteq Y$ : nonempty, closed (and convex) $\}$. A multifunction (setvalued function), $F: T \rightarrow \stackrel{f}{\rightarrow}(Y)$ is said to be measurable if for all $x \in Y$, the $\mathbb{R}_{+}$-valued function $t \rightarrow d(x, F(t))=\inf \{\|x-y\|: y \in F(t)\}$ is measurable. By $S_{F}^{p}(1 \leq p \leq \infty)$, we will denote the set of selectors of $F(\cdot)$ that belong to the Lebesgue-Bochner space $L^{p}(Y)$; i.e. $S_{F}^{p}=\left\{f \in L^{p}(Y)\right.$ : $f(t) \in F(t)$ a.e. $\}$. It is easy to check using Aumann's selection theorem (see for example Wagner [8], theorem 5.10), that $S_{F}^{p}$ is nonempty if and only if the $\mathbb{R}_{+}$-valued function $t \rightarrow \inf \{\|x\|: x \in F(t)\}$ belongs to $L_{+}^{p}$.

\footnotetext{
${ }^{1}$ Presently on leave at the Florida Institute of Technology, Department of Applied Mathematics, 150 West University Boulevard, Melbourne, Florida, 329091-6988, U.S.A.
} 
Let $H$ be a separable Banach space and $X$ a dense subspace of $H$, carrying the structure of a separable, reflexive Banach space, which embeds in $H$ continuously. Identifying $H$ with its dual (pivot space), we have $X \rightarrow H \rightarrow X^{*}$, with all embeddings being continuous and dense. Such a triple of spaces is known in the literature as "evolution triple" (or "Gelfand triple" or "spaces in normal position"). We will also assume that the above embeddings are compact, a condition that is very often satisfied in applications. By $\|\cdot\|\left(\right.$ resp. $\left.|\cdot|,\|\cdot\|_{*}\right)$, we will denote the norm of $X$ (resp. of $\left.H, X^{*}\right)$. Also by $\langle\cdot, \cdot\rangle$ we will denote the duality brackets for the pair $\left(X, X^{*}\right)$ and by $(\cdot, \cdot)$ the inner product of $H$. The two are compatible in the sense that $\left.\langle\cdot, \cdot\rangle\right|_{X \times H}=(\cdot, \cdot)$. To have a concrete example in mind let $Z \subseteq \mathbb{R}^{N}$ be a bounded domain, $X=W_{0}^{m, p}(Z), H=$ $L^{2}(Z)$ and $X^{*}=W_{0}^{m, p}(Z)^{*}=W^{-m, q}(Z), 2 \leq p<\infty, \frac{1}{p}+\frac{1}{q}=1$. From the well-known Sobolev's embedding theorem we know that $\left(X, H, X^{*}\right)$ is an evolution triple and furthermore all embeddings are compact. Let $W(T)=\left\{x \in L^{2}(X): \dot{x} \in L^{2}\left(X^{*}\right)\right\}$. The derivative in this definition is taken in the sense of vector valued distributions. Equipped with the norm $\|x\|_{W(T)}=$ $\left[\|x\|_{L^{2}(X)}^{2}+\|\dot{x}\|_{L^{2}\left(X^{*}\right)}\right]^{1 / 2}, W(T)$ becomes a separable reflexive Banach space. Furthermore if $X$ is a Hilbert space, then $W(T)$ is too, with inner product $(x, y)_{W(T)}=(x, y){ }_{L^{2}(X)}+$ $(\dot{x}, \dot{y})_{L^{2}\left(X^{*}\right)}, x, y \in W(T)$. Note that the elements in $W(T)$ are up to a Lebesgue-null subset of $T$, equal to an $X^{*}$-valued absolutely continuous function, and, therefore the derivative $\dot{x}(\cdot)$, is also the strong derivative of the function $x: T \rightarrow X^{*}$. Also, it is well-known that $W(T)$ embeds continuously into $C(T, H)$. Thus, every equivalence class in $W(T)$, has a unique representative in $C(T, H)$. Furthermore, since we have assumed that $X \rightarrow H$ compactly, we have that $W(T) \rightarrow L^{2}(H)$ compactly. Recently, Nagy [3] proved that if $X$ is a Hilbert space too, then $W(T) \rightarrow C(T, H)$ compactly. For further details on evolution triples and the abstract Sobolev space $W(T)$ we refer to the book of Zeidler [9] and, in particular, chapter 23.

Let $Z$ and $V$ be Hausdorff topological spaces. A multifunction $G: Z \rightarrow 2^{V} \backslash\{\emptyset\}$ is said to be upper semicontinuous (u.s.c.) (resp. lower semicontinuous (l.s.c.)), if for every open set $U \subseteq V$, the set $G^{+}(U)=\{z \in Z: G(z) \subseteq U\}$ (resp. the set $G^{-}(U)=\{z \in Z: G(z) \cap U \neq \emptyset\}$ ) is open in $Z$. Other equivalent definitions and further properties of such multifunctions can be found in the book of Klein-Thompson [2].

\section{Existence Theorems}

Let $T=[0, r]$ and $\left(X, H, X^{*}\right)$ be an evolution triple of spaces with all embeddings assumed to be compact. We will be considering the following second order nonlinear evolution inclusion:

$$
\left\{\begin{array}{c}
\ddot{x}(t)+A(t, \dot{x}(t))+B x(t) \in F(t, x(t)) \text { a.e. } \\
x(0)=x_{0} \in X, \dot{x}(0)=x_{1} \in H .
\end{array}\right\}
$$

By a solution of $(*)$, we understand a function $x \in C(T, X)$ such that $\dot{x} \in W(T)$ and an $f \in S_{F(\cdot, x(\cdot))}^{2}$ such that $\ddot{x}(t)+A(t, \dot{x}(t))+B x(t)=f(t)$ a.e. with $x(0)=x_{0}$ and $\dot{x}(0)=x_{1}$. Recall (see Section 2), that $W(T) \rightarrow C(T, H)$ and so the initial condition $\dot{x}(0)=x_{1} \in H$ makes sense.

First we prove an existence theorem for $(*)$, for the case where the multivalued perturbation term $F(t, x)$ is convex-valued. To this end, we will need the following hypotheses on the data of $(*)$. 
$H(A): \quad A: T \times X \rightarrow X^{*}$ is a map such that

(1) $\quad t \rightarrow A(t, v)$ is measurable,

(2) $v \rightarrow A(t, v)$ is monotone, hemicontinuous (i.e. for all $v, v^{\prime} \in X,\left\langle A(t, v)-A\left(t, v^{\prime}\right)\right.$, $\left.v-v^{\prime}\right\rangle \geq 0$ (monotonicity) and for all vectors $v, y, x \in X$, the map $\lambda \rightarrow\langle A(t, v+\lambda y), x\rangle$ is continuous on $[0,1]$ (demicontinuity)),

(3) $\quad\langle A(t, v), v\rangle \geq c\|v\|^{2}$ a.e. with $c>0$,

(4) $\quad\|A(t, v)\|_{*} \leq a(t)+b\|v\|$ a.e. with $a(\cdot) \in L_{+}^{2}, b>0$.

$H(B): \quad B \in \mathcal{L}\left(X, X^{*}\right), \quad\langle B x, y\rangle=\langle x, B y\rangle \quad$ for $\quad$ all $x, y \in X \quad$ (i.e. $B$ is symmetric) and $\langle B x, x\rangle \geq c^{\prime}\|x\|^{2}$ with $c^{\prime}>0$.

$H(F)_{1}: \quad F: T \times H \rightarrow P_{f c}(H)$ is a multifunction such that

(1) $\quad t \rightarrow F(t, x)$ is measurable,

(2) $\quad x \rightarrow F(t, x)$ is u.s.c. from $H$ into $H_{w}$,

() $\quad|F(t, x)|=\sup \{|v|: v \in F(t, x)\} \leq a_{1}(t)+b_{1}|x|$ a.e. with $a_{1}(\cdot) \in L_{+}^{2}, b_{1}>0$.

We will denote the solution set of $(*)$ by $S\left(x_{0}, x_{1}\right) \subseteq C(T, X)$.

Theorem 3.1: If hypotheses $H(A), H(B), H(F)_{1}$ hold and $x_{0} \in X, x_{1} \in H$, then $S\left(x_{0}, x_{1}\right)$ is a nonempty and compact subset of $C(T, X)$.

Proof: First we will derive some a priori bounds for the solutions of $(*)$. Let $x(\cdot) \in$ $C(T, X)$ be such a solution. Then, by the definition, for some $f \in S_{F(\cdot, x(\cdot))}^{2}$, we have

$$
\ddot{x}(t)+A(t, \dot{x}(t))+B(x(t))=f(t) \text { a.e. }
$$

it yields

$$
\langle\ddot{x}(t), \dot{x}(t)\rangle+\langle A(t, \dot{x}(t)), \dot{x}(t)\rangle+\langle B x(t), \dot{x}(t)\rangle=(f(t), \dot{x}(t)) \text { a.e. }
$$

Since $\dot{x} \in W(T)$, from proposition 23.23 (iv), p. 422 of Zeidler [9], we know that

$$
\langle\ddot{x}(t), \dot{x}(t)\rangle=\frac{1}{2} \frac{d}{d t}|\dot{x}(t)|^{2} .
$$

Also because of hypothesis $H(A)(\underline{3})$, we have that

$$
\langle A(t, \dot{x}(t)), \dot{x}(t)\rangle \geq c\|\dot{x}(t)\|^{2} \text { a.e. }
$$

Using the product rule and the symmetry hypothesis on $B$, we get

$$
\begin{aligned}
\frac{d}{d t}\langle B x(t), x(t)\rangle & =\langle B \dot{x}(t), x(t)\rangle+\langle B x(t), \dot{x}(t)\rangle \\
& =2\langle B x(t), \dot{x}(t)\rangle .
\end{aligned}
$$

Substituting (2), (3) and (4) into (1) above, we finally have

$$
\frac{1}{2} \frac{d}{d t}|\dot{x}(t)|^{2}+c\|\dot{x}(t)\|^{2}+\frac{1}{2} \frac{d}{d t}\langle B x(t), x(t)\rangle \leq(f(t), \dot{x}(t)) \text { a.e. }
$$

Integrating the above inequality, we get that

$$
\frac{1}{2}|\dot{x}(t)|^{2}-\frac{1}{2}\left|x_{1}\right|^{2}+c \int_{0}^{t}\|\dot{x}(s)\|^{2} d s+\frac{1}{2}\langle B x(t), x(t)\rangle-\frac{1}{2}\left\langle B x_{0}, x_{0}\right\rangle \leq \int_{0}^{t}(f(s), \dot{x}(s)) d s
$$


it yields

$$
|\dot{x}(t)|^{2}+2 c \int_{0}^{t}\|\dot{x}(s)\|^{2} d s+c^{\prime}\|x(t)\|^{2} \leq M+2 \int_{0}^{t}(f(s), \dot{x}(s)) d s
$$

where $M=\left|x_{1}\right|^{2}+\|B\|_{\mathcal{L}}\left\|x_{0}\right\|^{2}$.

Applying Cauchy's inequality with $\epsilon>0$, we get

$$
\begin{gathered}
\int_{0}^{t}(f(s), \dot{x}(s)) d s \leq \int_{0}^{t}|f(s)| \cdot|\dot{x}(s)| d s \\
\leq \frac{\epsilon}{2} \int_{0}^{t}|f(s)|^{2} d s+\frac{1}{2 \epsilon} \int_{0}^{t}|\dot{x}(s)|^{2} d s \\
\leq \frac{\epsilon}{2} \int_{0}^{t}\left(2 a_{1}(s)^{2}+2 b_{1}^{2}|x(s)|^{2}\right) d s+\frac{1}{2 \epsilon} \int_{0}^{t}|\dot{x}(s)|^{2} d s \\
\leq \epsilon \int_{0}^{t}\left(a_{1}(s)^{2}+b_{1}^{2}|x(s)|^{2}\right) d s+\frac{1}{2 \epsilon} \int_{0}^{t} \beta^{2}\|\dot{x}(s)\|^{2} d s
\end{gathered}
$$

where $\beta>0$ is such that $|\cdot| \leq \beta\|\cdot\|$. It exists since by hypothesis $X \rightarrow H$ continuously. So, we have

$$
\begin{gathered}
|\dot{x}(t)|^{2}+2 c \int_{0}^{t}\|\dot{x}(s)\|^{2} d s+c^{\prime}\|x(t)\|^{2} \\
\leq M+\epsilon\left\|a_{1}\right\|_{2}^{2}+\epsilon b_{1}^{2} \int_{0}^{t}|x(s)|^{2} d s+\frac{\beta^{2}}{2 \epsilon} \int_{0}^{t}\|\dot{x}(s)\|^{2} d s .
\end{gathered}
$$

Let $\frac{\beta^{2}}{2 \epsilon}=2 c$ implies that $\epsilon=\frac{\beta^{2}}{4 c}$. Then we have:

$$
|\dot{x}(t)|^{2}+\frac{c^{\prime}}{\beta^{2}}|x(t)|^{2} \leq M+\frac{\beta^{2}}{4 c}\left\|a_{1}\right\|_{2}^{2}+\frac{\beta^{2}}{4 c} b_{1}^{2} \int_{0}^{t}|x(s)|^{2} d s .
$$

From (*) by neglecting $|\dot{x}(t)|^{2}$ and using Gronwall's inequality, we get

$$
|x(t)|^{2} \leq\left(\frac{\beta^{2}}{c^{\prime}} M+\frac{\beta^{4}}{4 c c^{\prime}}\left\|a_{1}\right\|_{2}^{2}\right) \exp \left(\frac{\beta^{2} b_{1}^{2}}{4 c c^{\prime}} r\right)=M_{2}^{2}, t \in T
$$

Using (6) and neglecting $\frac{c^{\prime}}{\beta^{2}}|x(t)|^{2}$ in $(*)$, we obtain 


$$
|\dot{x}(t)|^{2} \leq M+\frac{\beta^{2}}{4 c}\left\|a_{1}\right\|_{2}^{2}+\frac{\beta^{2}}{4 c} b_{1}^{2} M_{2}^{2} r=M_{1}^{2}, t \in T
$$

Coming back to (5) and using estimates (6) and (7) above, we get

$$
\|\dot{x}\|_{L^{2}(X)} \leq \frac{1}{2 c}\left(M+2\left\|a_{1}\right\|_{2}^{2}+M_{2}^{2} r+M_{1}^{2} r\right)=M_{3}^{2} .
$$

Finally, from (5) and (8), we deduce that

$$
\|x(t)\|^{2} \leq \frac{1}{c^{\prime}}\left(M+2\left\|a_{1}\right\|_{2}^{2}+2 b_{1}^{2} M_{2}^{2} r+M_{1}^{2} r\right)=M_{4}^{2} .
$$

Finally, let $p \in L^{2}(X)$ and denote by $((\cdot, \cdot))_{0}$ the duality brackets for the pair $\left(L^{2}(X)\right.$, $\left.L^{2}\left(X^{*}\right)=L^{2}(X)^{*}\right)$. Also let $\widehat{A}: L^{2}(X) \rightarrow L^{2}\left(X^{*}\right)$ be the Nemitsky operator corresponding to the map $A(t, x)$; i.e. $(\widehat{A} x)(t)=A(t, x(t))$. Then we have:

$$
\begin{gathered}
((\ddot{x}, p))_{0} \leq\left|((\widehat{A}(\dot{x}), p))_{0}\right|+\left|((B x, p))_{0}\right|+((f, p))_{0} \\
\leq\left[\|\widehat{A}(x)\|_{L^{2}\left(X^{*}\right)}+\|B x\|_{L^{2}\left(X^{*}\right)}+\|f\|_{L^{2}\left(X^{*}\right)}\right]\|p\|_{L^{2}(X)} \\
\leq\left[\|a\|_{2}+b M_{3}+\|B\|_{\mathcal{L}} M_{r} r^{1 / 2}+\beta^{\prime}\left\|a_{1}\right\|_{2}+\beta^{\prime} b_{1} M_{2} r^{1 / 2}\right]\|p\|_{L^{2}(X)}
\end{gathered}
$$

where $\beta^{\prime}>0$ is such that $\|\cdot\|_{*} \leq \beta^{\prime}|\cdot|$. It exists since $H \rightarrow X^{*}$ continuously. Since $p \in L^{2}(X)$ was arbitrary, we deduce that there exists $M_{5}>0$ such that for all $x \in S\left(x_{0}, x_{1}\right)$, we have

$$
\|\ddot{x}\|_{L^{2}\left(X^{*}\right)} \leq M_{5} \text {. }
$$

From (8) and (10) above, we deduce that the set

$$
S^{\prime}\left(x_{0}, x_{0}\right)=\left\{\dot{x} \in W(T): x \in S\left(x_{0}, x_{1}\right)\right\}
$$

is bounded, hence relatively weakly compact in $W(T)$.

Now introduce the following modification of the original orientor field $F(t, x)$ :

$$
\widehat{F}(t, x)=\left\{\begin{array}{cc}
F(t, x) & \text { if }|x| \leq M_{2} \\
F\left(t, \frac{M_{2} x}{|x|}\right) & \text { if }|x|>M_{2}
\end{array}\right.
$$

Observe that $\widehat{F}(t, x)=F\left(t, p_{M_{2}}(x)\right)$, where $p_{M_{2}}(\cdot)$ is the $M_{2}$-radial retraction in $H$. Since $p_{M_{2}}(\cdot)$ is Lipschitz continuous, we have, using hypothesis $H(F)_{1}$, that $t \rightarrow \widehat{F}(t, x)$ is measurable while $x \rightarrow \widehat{F}(t, x)$ is u.s.c. from $H$ into $H_{w}$. Furthermore, note that $|\widehat{F}(t, x)| \leq a(t)+b M_{2}=\phi(t)$ a.e., with $\phi(\cdot) \in L_{+}^{2}$. Let $K=\left\{h \in L^{2}(H): h(t) \mid \leq \phi(t) a . e.\right\}$. This set, endowed with the relative weak $L^{2}(H)$-topology, is compactly metrizable. In what follows, this will be the topology considered on $K$. Let $\gamma: K \rightarrow C(T, X)$ be the map which to each $h \in K$, assigns the unique solution of the initial value problem $\ddot{x}(t)+A(t, \dot{x}(t))+B x(t)=h(t), x(0)=x_{0}, \dot{x}(0)=x_{1}$ (see Zeidler [9], theorem 33.A, p. 224). We claim that $\gamma(\cdot)$ is continuous. To this end, let $h_{n} \rightarrow h$ in $K$ and let $x_{n}=\gamma\left(h_{n}\right)$. Recall that $\left\{\dot{x}_{n}\right\}_{n>1} \subseteq W(T)$ is relatively weakly compact. Hence, by passing to a subsequence if necessary, we may assume that $\dot{x}_{n} \stackrel{w}{\rightarrow} y$ in $W(T)$. Let $x=\gamma(h)$. We need to show that $y=\widehat{x}$. We have: 


$$
\begin{aligned}
\left\langle\ddot{x}_{n}(t)-\ddot{x}(t), \dot{x}_{n}(t)-\dot{x}(t)\right\rangle+\left\langle A\left(t, \dot{x}_{n}(t)\right)-A(t, \dot{x}(t)), \dot{x}_{n}(t)-\dot{x}(t)\right\rangle \\
+\left\langle B x_{n}(t)-B x(t), \dot{x}_{n}(t)-\dot{x}(t)\right\rangle \\
=\left(h_{n}(t)-h(t), \dot{x}_{n}(t)-\dot{x}(t)\right) \text { a.e. }
\end{aligned}
$$

Exploiting the fact that $A(t, \cdot)$ is monotone and using the integration by parts formula for functions in $W(T)$ (see Zeidler [9], proposition 23.23, p. 422), we get

$$
\frac{1}{2} \frac{d}{d t}\left|\dot{x}_{n}(t)-\dot{x}(t)\right|^{2}+\left\langle B\left(x_{n}(t)-x(t)\right), \dot{x}_{n}(t)-\dot{x}(t)\right\rangle \leq\left(h_{n}(t)-h(t), \dot{x}_{n}(t)-\dot{x}(t)\right) \text { a.e. }
$$

But, as before, exploiting the symmetry of the operator $B$, we have

$$
\left\langle B\left(x_{n}(t)-x(t)\right), \dot{x}_{n}(t)-\dot{x}(t)\right\rangle=\frac{1}{2} \frac{d}{d t}\left\langle B\left(x_{n}(t)-x(t)\right), x_{n}(t)-x(t)\right\rangle .
$$

So we get:

$$
\frac{1}{2} \frac{d}{d t}\left|\dot{x}_{n}(t)-\dot{x}(t)\right|^{2}+\frac{1}{2} \frac{d}{d t}\left\langle B\left(x_{n}(t)-x(t)\right), x_{n}(t)-x(t)\right\rangle \leq\left(h_{n}(t)-h(t), \dot{x}_{n}(t)-\dot{x}(t)\right) \text { a.e. }
$$

Integrating and recalling that $x_{n}(0)=x(0)=x_{0}, \dot{x}_{n}(0)=\dot{x}(0)=x_{1}$, we have:

$$
\frac{1}{2}\left|\dot{x}_{n}(t)-\dot{x}(t)\right|^{2}+\frac{1}{2}\left\langle B\left(x_{n}(t)-x(t)\right), x_{n}(t)-x(t)\right\rangle \leq \int_{0}^{t}\left(h_{n}(s)-h(s), \dot{x}_{n}(s)-\dot{x}(s)\right) d s
$$

which yields

$$
\frac{c^{\prime}}{2}\left\|x_{n}(t)-x(t)\right\|^{2} \leq \int_{0}^{t}\left(h_{n}(s)-h(s), \dot{x}_{n}(s)-\dot{x}(s)\right) d s
$$

which yields

$$
\left\|x_{n}(t)-x(t)\right\|^{2} \leq \frac{2}{c^{\prime}} \int_{0}^{t}\left(h_{n}(s)-h(s), \dot{x}_{n}(s)-\dot{x}(s)\right) d s .
$$

Note that $h_{n} \stackrel{w}{\rightarrow} h$ in $L^{2}(H)$ and $\dot{x}_{n} \stackrel{w}{\rightarrow} y$ in $W(T)$. Since $W(T) \rightarrow L^{2}(H)$ compactly, we have that $\dot{x}_{n} \stackrel{s}{\rightarrow} y$ in $L^{2}(H)$. Thus we have:

$$
\begin{gathered}
\int_{0}^{t}\left(h_{n}(s)-h(s), \dot{x}_{n}(s)-\dot{x}(s)\right) d s \\
=\int_{0}^{t}\left(h_{n}(s)-h(s), \dot{x}_{n}(s)-y(s)\right) d s+\int_{0}^{t}\left(h_{n}(s)-h(s), y(s)-\dot{x}(s)\right) d s \rightarrow 0 \text { as } n \rightarrow \infty .
\end{gathered}
$$

So $x_{n}(t) \stackrel{s}{\rightarrow} x(t)$ in $X$ yields $\dot{x}=y \in W(T)$. Now note that

$$
\left\|x_{n}(t)-x(t)\right\|^{2} \leq \frac{2}{c^{\prime}}\left\|h_{n}-h\right\|_{L^{2}(H)}\left\|\dot{x}_{n}-\dot{x}\right\|_{L^{2}(H)} .
$$

Since $h_{n} \stackrel{w}{\rightarrow} h$ in $K$, we have $\left\|h_{n}-h\right\|_{L^{2}(H)} \leq N$ for all $n \geq 1$ and some $N>0$. Thus 


$$
\left\|x_{n}(t)-x(t)\right\|^{2} \leq \frac{2}{c^{\prime}} N\left\|\dot{x}_{n}-\dot{x}\right\|_{L^{2}(H)} \rightarrow 0
$$

which implies that $\gamma(\cdot)$ is indeed continuous as claimed.

Let $R: K \rightarrow 2^{K}$ be the multifunction defined by

$$
R(h)=S_{\widehat{F}(\cdot, \gamma(h)(\cdot))}^{2} \cdot
$$

First we will show that $R(\cdot)$ has nonempty values. Let $s_{n}(\cdot)$ be simple functions such that $s_{n}(t) \stackrel{s}{\rightarrow} \gamma(h)(t)$ a.e. in $H$. Then because of hypothesis $H(F)_{1}(\underline{1})$, for each $n \geq 1, t \rightarrow \widehat{F}\left(t, s_{n}(t)\right)$ is measurable. Apply Aumann's selection theorem to get $f_{n}: T \rightarrow H$ measurable such that $f_{n}(t) \in$ $\widehat{F}\left(t, x_{n}(t)\right)$ a.e., $n \geq 1$. Note that $\left|f_{n}(t)\right| \leq \phi(t)$ a.e. with $\phi(\cdot) \in L_{+}^{2}$. Hence by passing to a subsequence if necessary, we may assume that $f_{n} \stackrel{w}{\rightarrow} f$ in $L^{2}(H)$. Then theorem 3.1 of [6], tells us that

$$
\begin{gathered}
f(t) \in \overline{\overline{c o n v}} w-\overline{\lim }\left\{f_{n}(t)\right\}_{n \geq 1} \\
\subseteq \overline{\operatorname{conv}} w-\overline{\lim } \widehat{F}\left(t, s_{n}(t)\right) \\
\subseteq \widehat{F}(t, \gamma(t)(t)) \text { a.e. }
\end{gathered}
$$

The last inclusion follows from the fact that $\widehat{F}(t, \cdot)$ is $u . s . c$. from $H$ into $H_{w}$ and since $s_{n}(t) \stackrel{s}{\rightarrow}$ $\gamma(h)(t)$ a.e. in $H$. Therefore $f \in S_{\widehat{F}(\cdot, p(h)(\cdot))}^{2}$ and so we have established that the values of the multifunction $R(\cdot)$ are nonempty. Also since $F(t, x)$ is $P_{f c}(H)$-valued, it is clear that for every $h \in K, \quad R(h) \in P_{f c}(K)$. Furthermore using theorem 4.2 of [6] and recalling that $\gamma(\cdot)$ is continuous on $K$ into $C(T, X)$, we get that $R(\cdot)$ is u.s.c. Apply the Kakutani-KyFan fixed point theorem to get $h \in R(h)$. Then $x=\gamma(h)$ is a solution of $(*)$, with $F(t, x)$ replaced by $\widehat{F}(t, x)$. But as in the beginning of the proof, with the same a priori estimation, we can show that $|x(t)| \leq M_{2}$ for all $t \in T$ implies that $\widehat{F}(t, x(t))=F(t, x(t))$ and this yields that $x(\cdot)$ solves $(*)$.

Finally to establish the compactness of $S\left(x_{0}, x_{1}\right)$ in $C(T, X)$, note that $S\left(x_{0}, x_{1}\right) \subseteq \gamma(K)$ and the latter is compact in $C(T, X)$ since $\gamma: K \rightarrow C(T, X)$ is continuous. So it suffices to show that $S\left(x_{0}, x_{1}\right)$ is closed in $C(T, X)$. So let $\left\{x_{n}\right\}_{n \geq 1} \subseteq S\left(x_{0}, x_{1}\right)$ and assume that $x_{n} \rightarrow x$ in $C(T, X)$. Then by definition $x_{n}=\gamma\left(f_{n}\right)$ with $f_{n} \in S_{F(\cdot)\left(x_{n}(\cdot)\right)}^{2}$. Note that because of hypothesis $H(F)_{1}(\underline{3})$ $\left|f_{n}(t)\right| \leq a_{1}(t)+b_{1} \widehat{N}$, where $\widehat{N}=\sup \left\|x_{n}\right\|_{C(T, X)}$. So we may assume that $f_{n} \stackrel{w}{\rightarrow} f$ in $L^{2}(H)$ implies that $\gamma\left(f_{n}\right) \rightarrow \gamma(f)$ in $C(T, X)$ which yields $x=\gamma(f)$ and from theorem 3.1 of [6], we have

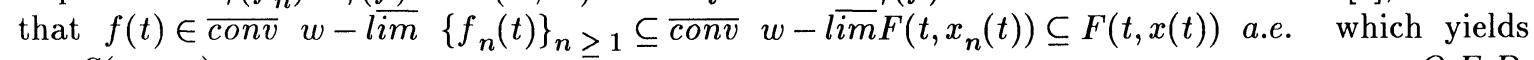
$x \in S\left(x_{0}, x_{1}\right)$.

Q.E.D.

Now we consider the case where the multivalued perturbation term $F(t, x)$ is not necessarily convex-valued. We will need the following hypothesis on the orientor field $F(t, x)$.

$\underline{H\left(F_{2}\right)}: \quad F: T \times H \rightarrow P_{f}(H)$ is a multifunction such that

(1) $\quad(t, x) \rightarrow F(t, x)$ is graph measurable; i.e. $G r F=\{(t, x, y) \in T \times H \times H: y \in$ $F(t, x)\} \in B(T) \times B(H)$, with $B(T)$ (resp. $B(H)$ ), being the Borel $\sigma$-field of $T$ (resp. of $H$ ) (recall that measurability of $F(\cdot, \cdot)$ implies graph measurability).

(2) $\quad x \rightarrow F(t, x)$ is l.s.c.

() $\quad|F(t, x)|=\sup \{|y|: y \in F(t, x)\} \leq a_{1}(t)+b_{1}|x|$ a.e. with $a_{1}(\cdot) \in L_{+}^{2}$, $b_{1}>0$.

Theorem 3.2: If hypotheses $H(A), H(B), H(F)_{2}$ hold and $x_{0} \in X, x_{1} \in H$, then $S\left(x_{0}, x_{1}\right) \neq$ $\emptyset$. 
Proof: As in the proof of theorem 3.1, let $\widehat{F}(t, x)=F\left(t, p_{M_{2}}(x)\right)$ (it is clear that the same $a$ priori estimation is valid in the present situation). Then given that $p_{M_{2}}(\cdot)$ is Lipschitz continuous, we have that $(t, x) \rightarrow \widehat{F}(t, x)$ is graph measurable, $x \rightarrow \widehat{F}(t, x)$ is l.s.c. and furthermore note that $|\widehat{F}(t, x)| \leq a_{1}(t)+b_{1} M_{2}=\phi(t)$ a.e. with $\phi(\cdot) \in L_{+}^{2}$.

Let $V \subseteq L^{1}(H)$ be defined by $V=\left\{h \in L^{1}(H):|h(t)| \leq \phi(t)\right.$ a.e. $\}$. From proposition 3.1 of [5], we know that $V$, equipped with the relative weak $L^{1}(H)$-topology, is compact metrizable. Consider the multifunction $\Gamma: V \rightarrow P_{f}\left(L^{1}(H)\right)$ defined by $\Gamma(h)=S_{\widehat{F}(\cdot, \gamma(h)(\cdot))}^{1}$. It is easy to check using the continuity of $\gamma(\cdot)$ and theorem 4.1 of [6], that $\Gamma(\cdot)$ is l.s.c. ( note that if $h_{n} \stackrel{w}{\rightarrow} h$ in $V \subseteq L^{1}(H)$, then $h_{n} \stackrel{w}{\rightarrow} h$ in $L^{2}(H)$, since $\left.\phi(\cdot) \in L_{+}^{2}\right)$. So, we can apply Fryszkowski's continuous selection theorem [1], to get $k: V \rightarrow V$ continuous such that $k(h) \in R(h)$. Applying the SchauderTichonov fixed point theorem, we get $h \in V$ such that $h=k(h)$. Then $x=p(h)$ solves (*) with $F(t, x)$ replaced by $\widehat{F}(t, x)$. But as before we can check that $|x(t)| \leq M_{2}$ which implies $\widehat{F}(t, x(t))$ implies that $F(t, x(t))$ which yields $x \in S\left(x_{0}, x_{1}\right)$.

Q.E.D.

\section{An Example}

In this section we present an example of a nonlinear hyperbolic partial differential inclusion illustrating the applicability of our work.

So let $T=[0, r]$ and $Z$ a bounded domain in $\mathbb{R}^{N}$, with smooth boundary $\Gamma=\partial Z$. We will consider the following initial-boundary value problem of hyperbolic type with multivalued terms.

$$
\left\{\begin{array}{c}
\frac{\partial^{2} x}{\partial t^{2}}-\Delta x-\sum_{i=1}^{N} D_{i}\left(k\left(t,\left|D x_{t}\right|^{2}\right) D_{i} x_{t}\right) \in\left[f_{1}(t, z, x(t, z)), f_{2}(t, z, x(t, z))\right] \\
\left.x\right|_{T \times \Gamma}=0, x(0, z)=x_{0}(z), x_{t}(0, z)=x_{1}(z) .
\end{array}\right\}
$$

Here $\quad D_{i}=\frac{\partial}{\partial z_{i}} \quad i=1, \ldots, N, \quad D x=\left(D_{1} x_{1}, \ldots, D_{N} x\right)=\operatorname{grad}(x), \quad D x D y=\sum_{i=1}^{N} D_{i} x D_{i} y \quad$ and $|D x|^{2}=\sum_{i=1}^{N}\left|D_{i} x\right|^{2}$

We will need the following hypotheses on the data of $(* *)$ :

$\underline{H(k):} \quad k: T \times \mathbb{R}_{+} \rightarrow \mathbb{R}_{+}$is a function such that

(1) $\quad t \rightarrow k(t, \mu)$ is measurable,

(2) $\quad \mu \rightarrow k(t, \mu)$ is continuous,

() $\quad 0 \leq k\left(t, \lambda^{2}\right) \leq L$ for all $(t, \lambda) \in T \times \mathbb{R}_{+}$, with $L>0$ and $k(t, 0)=0$,

(4) $\quad k\left(t, \lambda^{2}\right) \lambda-k\left(t, \mu^{2}\right) \mu \geq d(\lambda-\mu)$ for all $\lambda, \mu \in \mathbb{R}_{+}, \lambda \geq \mu$ and for some $d>0$.

$H(f): \quad f_{1}, f_{2}: T \times Z \times \mathbb{R} \rightarrow \mathbb{R}$ are measurable functions such that $x \rightarrow f_{1}(t, z, x),-f_{2}(t, z, x)$ are l.s.c. and $\left|f_{i}(t, z, x)\right| \leq a_{1}(t, z)+b_{1}(z)|x|$ a.e. $i=1,2$ with $a_{1}(\cdot, \cdot) \in L^{2}(T \times Z)$, $b_{1}(\cdot) \in L^{\infty}(Z)$ and $f_{1}<\bar{f}_{2}$.

$\underline{A_{0}}: \quad x_{0}(\cdot) \in H_{0}^{1}(Z), x_{1}(\cdot) \in L^{2}(Z)$.

In this case, $X=H_{0}^{1}(Z), \quad H=L^{2}(Z)$ and $X^{*}=H_{0}^{1}(Z)^{*}=H^{-1}(Z)$. We know that $\left(X, H, X^{*}\right)$ is an evolution triple with all embeddings being compact (Sobolev embedding theorem). Consider the following Dirichlet forms:

$$
a_{1}(t, x, y)=\int_{Z} \sum_{i=1}^{N} k\left(t,|D x|^{2}\right) D_{i} x D_{j} y d z=\int_{Z} k\left(t,|D x|^{2}\right) D x D y d z
$$

and

$$
a_{2}(x, y)=\int_{Z} \sum_{i=1}^{N} D_{i} x D_{i} y d z=\int_{Z} D x D y d z
$$


for all $x, y \in H_{0}^{1}(Z)$.

Using hypothesis $H(k)(\underline{3})$, we get

$$
\left|a_{1}(t, x, y)\right| \leq L\|x\|_{H_{0}^{1}(Z)}\|y\|_{H_{0}^{1}(Z)} .
$$

So there exists a nonlinear operator $A: T \times X \rightarrow X^{*}$ such that

$$
\langle A(t, z), y\rangle=a_{1}(t, x, y)
$$

From Fubini's theorem we have that $t \rightarrow a_{1}(t, x, y)$ is measurable which implies that $t \rightarrow A(t, x)$ is weakly measurable. But $H^{-1}(Z)$ is a separable Hilbert space. So the Pettis measurability theorem tells us that $t \rightarrow A(t, x)$ is measurable. Also if $x_{n} \rightarrow x$ in $H_{0}^{1}(Z)$, then by passing to a subsequence if necessary, we will have that $\left|D x_{n}(z)\right|^{2} \rightarrow|D x(z)|^{2}$ a.e. and since by hypothesis $H(k)(\underline{2}) k(t, \cdot)$ is continuous we have $k\left(t,\left|D x_{n}(z)\right|^{2}\right) \rightarrow k\left(t,|D x(z)|^{2}\right)$ for all $t \in T$ and almost all $z \in Z$. Also $D_{i} x_{n} \stackrel{s}{\rightarrow} D_{i} x$ in $L^{2}(Z)$. Thus $\int_{Z}^{n} k\left(t,\left|D x_{n}\right|^{2}\right) D x_{n} D y d z \rightarrow \int_{Z} k\left(t,|D x|^{2}\right) D x D y d z$ implies that $A\left(t, x_{n}\right) \stackrel{w}{\rightarrow} A(t, x)$ which yields $A(t, \cdot)$ is demicontinuous, this hemicontinuous. Also we have

$$
\langle A(t, x)-A(t, y), x-y\rangle=\int_{Z}\left(k\left(t,|D x|^{2}\right) D x-k\left(t,|D y|^{2}\right) D y\right)(D x-D y) d z .
$$

Then, because of hypothesis $H(k)(\underline{2})$ and lemma $25.26(\underline{b})$, p. 524 of Zeidler [9], we have

$$
\langle A(t, x)-A(t, y), x-y\rangle \geq c\|x-y\|_{H_{0}^{1}(Z)}^{2}, c>0
$$

which yields that $A(t, \cdot)$ is strongly monotone.

Also since $k(t, 0)=0$ (by hypothesis $H(k)(\underline{3})$ ), we have $A(t, 0)=0$ yields that $A(t, \cdot)$ is coercive; i.e., $\langle A(t, x), x\rangle \geq c\|x\|_{H_{0}^{1}(Z)}^{2}$. Thus, we satisfied hypothesis $H(A)$.

Next note that by the Cauchy-Schwartz inequality, we have

$$
\left|a_{2}(x, y)\right| \leq\|x\|_{H_{0}^{1}(Z)}\|y\|_{H_{0}^{1}(Z)} .
$$

So there exists a continuous linear operator $B: X \rightarrow X^{*}$ such that

$$
\langle B x, y\rangle=a_{2}(x, y) .
$$

Clearly $\langle B x, y\rangle=\langle x, B y\rangle$; i.e. $B$ is symmetric and by Poincaré's inequality, we have $\langle B x, x\rangle \geq$ $c^{\prime}\|x\|_{H_{0}^{1}(Z)}^{2}, c^{\prime}>0$. Therefore, we satisfied hypothesis $H(B)$.

Next let $F: T \times L^{2}(Z) \rightarrow P_{f c}\left(L^{2}(Z)\right)$ be defined by

$$
F(t, x)=\left\{h \in L^{2}(Z): f_{1}(t, z, x(z)) \leq h(z) \leq f_{2}(t, z, x(z)) \text { a.e. }\right\} .
$$

Let $\eta: T \times Z \times \mathbb{R} \rightarrow P_{f c}(\mathbb{R})$ be defined by $\eta(t, z, x)=\left[f_{1}(t, z, x), f_{2}(t, z, x)\right]$. Because of hypothesis $H(f)$, we deduce that $\eta(\cdot, \cdot, \cdot)$ is measurable while $\eta(t, z, \cdot)$ is u.s.c. (see Klein-Thompson [2], p. 74). Note that $F(t, x)=S_{\eta(t, \cdot, x(\cdot))}^{2}$. So, from theorem 4.2 of [6], we have that $F(t, \cdot)$ is u.s.c. from $H$ into $H_{w}$, while clearly $t \rightarrow F(t, z)$ is measurable. Also, $|F(t, x)|=\sup \left\{|y|_{L^{2}(Z)}\right.$ : $y \in F(t, x)\} \leq \widehat{a}_{1}(t)+\widehat{b}_{1}|x|_{L^{2}(Z)}$, with $\widehat{a}_{1}(t)=\|a(t, \cdot)\|_{L^{2}(Z)}, \widehat{b}_{1}=\|b\|_{L^{\infty}(Z)}$. Thus, we satisfied hypothesis $H(F)_{1}$. Finally, let $\widehat{x}_{0}=x_{0}(\cdot) \in H_{0}^{1}(Z), \widehat{x}_{1}=x_{1}(\cdot) \in L^{2}(Z)$. 
Rewrite $(* *)$ in the following equivalent nonlinear evolution inclusion form:

$$
\left\{\begin{array}{c}
\dot{x}(t)+A(t, \dot{x}(t))+B x(t) \in F(t, x(t)) \\
x(0)=\widehat{x}_{0}, \dot{x}(0)=x_{1} .
\end{array}\right\}
$$

Theorem 4.1: If hypotheses $H(k), H(f)$ and $H_{0}$ hold, $\underline{\text { then }}(* *)$ has a solution $x \in$ $C\left(T, H_{0}^{1}(Z)\right)$ such that $\frac{\partial x}{\partial t} \in L^{2}\left(T, H_{0}^{1}(Z)\right) \cap C\left(T, L^{2}(Z)\right)$ and $\frac{\partial^{2} x}{\partial t^{2}} \in L^{2}\left(T, H^{-1}(Z)\right)$. Also, the solution set is compact in $C\left(T, H_{0}^{1}(Z)\right)$.

Now suppose that $(* *)$ corresponds to an optimal control problem; i.e.

$$
f_{1}(t, z, x)=f(t, z, x) u_{1}(z)
$$

and

$$
f_{2}(t, z, x)=f(t, z, x) u_{2}(z)
$$

with a function $f: T \times Z \times \mathbb{R} \rightarrow \mathbb{R}+$ such that $(t, z) \rightarrow f(t, z, x)$ is measurable, $x \rightarrow f(t, z, x)$ is continuous and $|f(t, z, x)| \leq a_{1}(t, z)+b_{1}(z) x$ a.e., with $a_{1}(\cdot, \cdot) \in L^{2}(T \times Z), b_{1}(\cdot) \in L^{\infty}(Z)$. The control constraint set is defined as

$$
U(t, z)=\left\{v \in \mathbb{R}: u_{1}(z) \leq v \leq u_{2}(z)\right\}
$$

with $0<u_{1}(z)<u_{2}(z) \leq M$ a.e.

We are also given a cost functional $J(x)=\int_{0}^{b} \int_{Z} L(t, z, x(t, z)) d z d t$ to be minimized over all ad missible trajectories. Assume that $L: T \times Z \times \mathbb{R} \rightarrow \overline{\mathbb{R}}=\mathbb{R} \cup\{+\infty\}$ is a measurable integrand such that $L(t, z, \cdot)$ is l.s.c. and $\phi(t, z)-M(z)|x| \leq L(t, z, x)$ a.e. with $\phi(\cdot, \cdot) \in L^{1}(T \times Z)$, $M(\cdot) \in L_{+}^{\infty}(Z)$. Then, $J(\cdot)$ is l.s.c. on $C\left(T, H_{0}^{1}(Z)\right)$, and so, using theorem 4.1 above, we deduce that this distributed parameter optimal control problem has a solution. Analogous results for parabolic systems can be found in [4].

\section{Acknowledgement}

The author would like to thank the referee for suggesting a way to simplify the proof of theorem 3.1 .

\section{References}

[1] Fryszkowski, A., Continuous selections for class of nonconvex multivalued maps, Studia Math. 76 (1983), 163-174.

[2] Klein, E. and Thompson, A., Theory of Correspondences, Wiley, New York 1984.

[3] Nagy, E., A theorem of compact embedding for functions with values in an infinite dimensional Hilbert space, Annales Univ. Sci. Budapest, Sectio Math. 23 (1980), 243-245.

[4] Papageorgiou, N.S., Existence of optimal controls for a class of nonlinear distributed parameter systems, Bull. Austr. Math. Soc. 43 (1991), 211-224.

[5] Papageorgiou, N.S., On the theory of Banach space valued integrable multifunctions. Part 1: Integration and conditional expectation, J. Multiv. Anal. 17 (1985), 185-206. 
[6] Papageorgiou, N.S., Convergence theorems for Banach space valued integrable multifunctions, Intern. J. Math. and Math. Sci. 10 (1987), 433-442.

[7] Papageorgiou, N.S., Continuous dependence results for a class of evolution inclusion, Proc. Edinburgh Math. Soc. 35 (1992), 139-158.

[8] Wagner, D., Survey of measurable selection theorems, SIAM J. Control and Optim. 15 (1977), 859-903.

[9] Zeidler, E., Nonlinear Functional Analysis and its Applications II, Springer Verlag, New York 1990. 


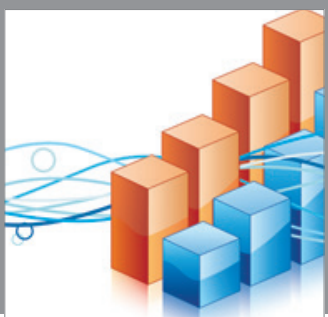

Advances in

Operations Research

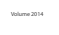

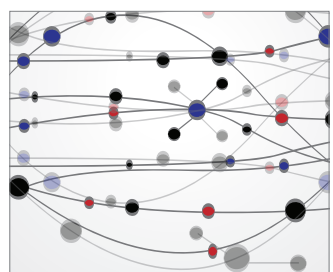

\section{The Scientific} World Journal
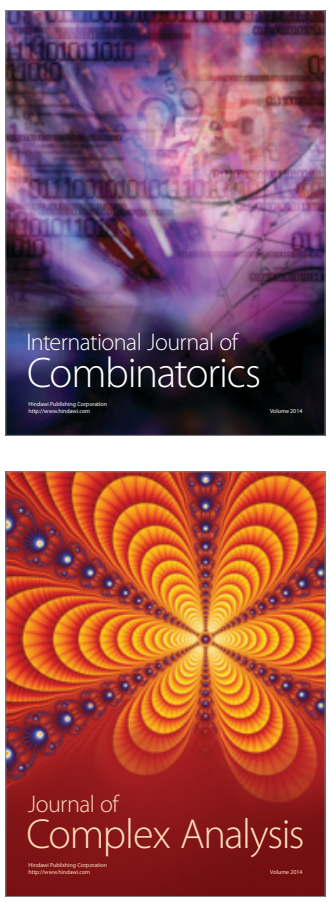

International Journal of

Mathematics and

Mathematical

Sciences
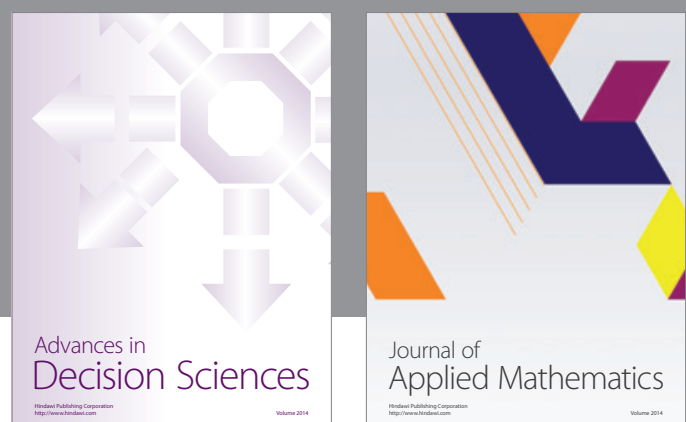

Journal of

Applied Mathematics
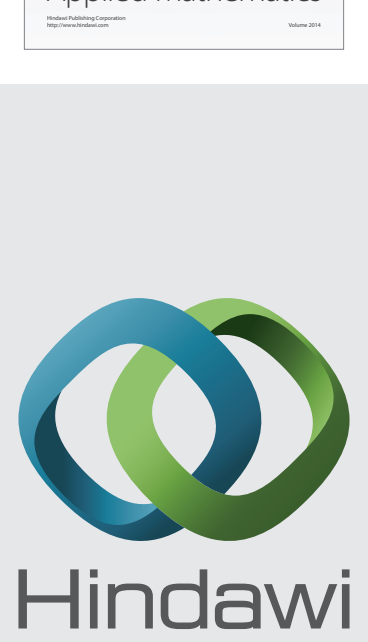

Submit your manuscripts at http://www.hindawi.com
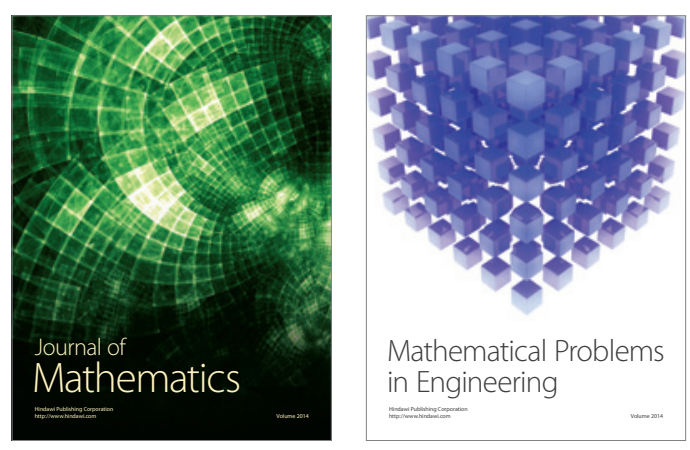

Mathematical Problems in Engineering
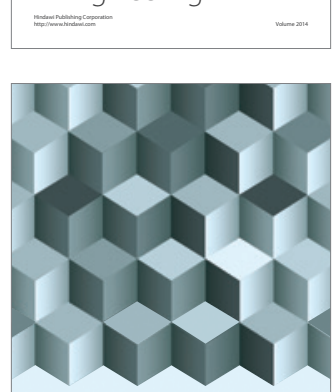

Journal of

Function Spaces
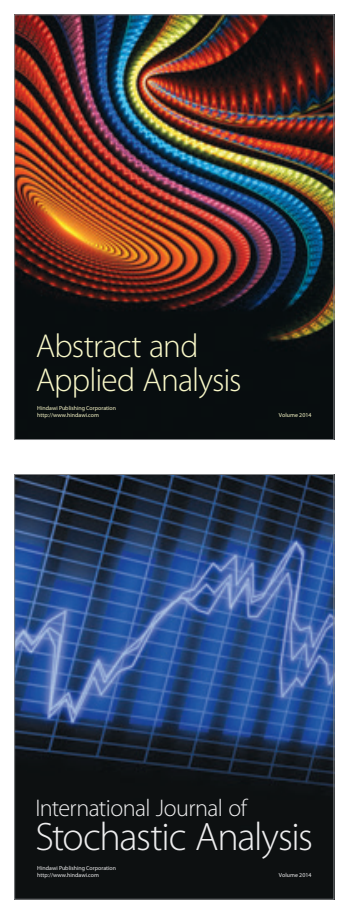

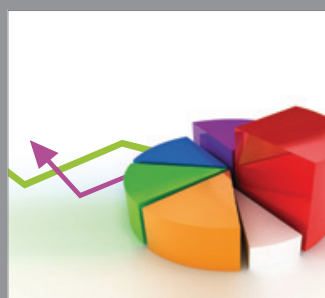

ournal of

Probability and Statistics

Promensencen
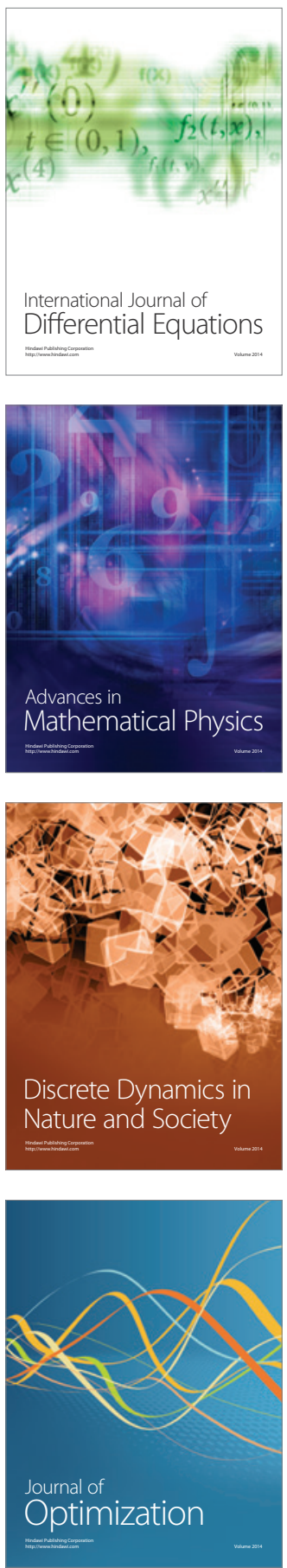